Article type : Special Issue Article

Corresponding Author Email ID: n.steinbeis@ucl.ac.uk

\title{
Editorial to the Special Issue on "On mechanisms of cognitive training and transfer in development"
}

\author{
Nikolaus Steinbeis \& Eamon McCrory
}

Division of Psychology and Language Sciences, UCL, London, WC1H OAP, UK

There is a popular appeal to the idea that learning to play an instrument could make one better at languages or that playing chess improves one's social skills. Does learning from experience generalize from one context to another? If so, what are the mechanisms that underpin such transferability? These questions are central to advancing our understanding of the extraordinary adaptability of the human species. They get at fundamental issues of our cognitive architecture and how we learn.

One of the most rigorous and experimentally controlled ways to address these questions are training studies. Here, one cognitive skill is repeatedly trained over several days or weeks and performance in other domains are assessed before and after the training to test for improvements. There is a wealth of literature using such paradigms (Strobach \& Karbach, 2016), spanning a considerable range of social, affective and cognitive functions. Yet whether interventions lead to effects beyond the domain directly trained remains controversial. While it is uncontested that interventions can impact closely related domains, so-called near transfer, it is unclear whether they also lead to improvements in more distantly related domains (Diamond \& Lee, 2011; Sala \& Gobet, 2019). To provide more clarity on these issues, there have been several recent best-practice recommendations (Green et al., 2019; Simons et al., 2016).

This article has been accepted for publication and undergone full peer review but has not been through the copyediting, typesetting, pagination and proofreading process, which may lead to differences between this version and the Version of Record. Please cite this article as doi: 10.1111/DESC.12932

This article is protected by copyright. All rights reserved 
However, a mechanistic account of how and why cognitive trainings work and lead to transfer effects remains outstanding.

Understanding how and why learning generalizes during early development is particularly relevant in light of the heightened behavioral and neural plasticity during this period, and the possibility that we might be able to promote cognitive functions critical for healthy psychological development (Johnson, 2011; Karmiloff-Smith, 1998; Wass, Scerif, \& Johnson, 2012). This Special Issue comprises a diverse set of empirical papers that illustrate some of the latest advances in our efforts to understand cognitive training and transfer in the context of child development. Given the controversy surrounding the issue of far transfer in cognitive training (Sala \& Gobet, 2019), scientific rigour in the empirical work is essential. Each study in this special issue adheres to best practice recommendations (Green et al., 2019; Simons et al., 2016). This creates the potential for significant insights into a range of functions and abilities, spanning academic achievement, language learning, executive functions and mental health and the mechanisms supporting transfer.

\section{Identifying mechanisms for training}

One crucial component in the logic of almost all training studies is an initial correlation between a training mechanism (i.e. digit span) and an outcome measure (i.e. fluid reasoning). What is often unclear and less examined is why such associations exist in the first place and especially at which level they operate (i.e. is it rehearsal, storage or retrieval of working memory that is related to IQ?). Addressing such basic questions is essential if we are to properly delineate suitable training mechanisms. Altarelli et al study how audio-visual associative learning, which is the basic process underlying learning to read, is related more generally to phonological awareness, language comprehension and executive function. Using a novel associative learning task of nonlinguistic auditory signals with novel visual shapes, the authors find that learning was strongly related to phonological awareness and language comprehension but not to executive functions. This highlights associative learning processes as a potential target for training phonological awareness and language comprehension.

\section{Training to excel: mechanisms underlying improved academic achievement}

Training paradigms in psychology are one of the few ways in which causal relationships between cognitive functions can be established. Typically, studies tend to focus on domains that are 
relevant for future life success (intelligence or academic achievement). This special issue includes several papers targeting a broad range of training mechanisms to improve academic achievement. There is ample evidence of a strong link between executive functions (i.e. working memory, cognitive flexibility, inhibition) and academic achievement (i.e. reading and maths; Best, Miller, \& Naglieri, 2011), and as a result there have been many attempts to train executive functions (EFs) as a way to improve reading and maths (Titz \& Karbach, 2014). Among these, working memory training has featured most prominently, yet results of academic improvements have been mixed (Karbach \& Verhaeghen, 2014; Sala \& Gobet, 2017). In this issue, Johann and Karbach sought to separately train working memory, flexibility and inhibition and test its effect on reading and maths. Importantly, each training occurred in a standard and a gamified version, which increased participant engagement. It was found that reading (but not maths) ability improved following game-based flexibility and inhibition training compared to a control group, with significant transfer at 3-month follow-up. These findings highlight the importance of executive functions other than working memory in improving academic achievement and demonstrate that engagement is a crucial moderating variable in whether durable transfer becomes evident.

There appears to be a relationship between mathematical skills and other cognitive skills such as spatial and musical abilities (Schellenberg, 2005; Uttal et al., 2013). Two training studies in this Special Issue capitalize on these links. Gilligan et al trained 250 8-year olds either on one of two types of spatial skills (i.e. mental rotation or spatial scaling), or a control training. They showed that both types of spatial skill training led to significant improvements on a range of mathematical abilities, including numerical representation, arithmetic and geometry. Azarzahu et al recruited 9year olds to test for the effectiveness of music-based interventions on fractions learning over standard teachings. This approach draws on the process-based links between the domains of music and maths (e.g. use of symbols, shared concept of parts making up a whole, the use of patterns). In two music programs, students learned to write musical notes and perform rhythmic patterns. This was used to produce factions and create addition/subtraction problems. The socalled "acoustic" approach focussed on musical notation and the temporal value of musical notes, whereas the "holistic" approach also included melodic features and included listening, playing and creating music on top of acoustic elements. In a comparison group, students were taught fractions in the regular manner set by the curriculum. It was found that musical fractions training was more effective than conventional approaches and this effect was even more evident for the holistic approach, for trained as well as untrained fractions and at 3- and 6-month follow-up.

What might be regarded as more 'direct' approaches of improving academic achievement are illustrated in this Special Issue in a study by Ramani et al with 6-year olds'. Here, the effects of 
training specific mathematical abilities (i.e. numerical magnitude knowledge) or general underlying cognitive skills (i.e. working memory) were evaluated in relation to a broad range of mathematical knowledge measures. Using a tablet-based training game, two training groups were compared with an active control group. Comparison occurred at a latent factor level derived from several measures of mathematical ability (e.g. counting, addition, numerical identification, number line, magnitude comparison). It was found that playing the numbers game improved children's numerical knowledge at the latent factor level and continued to do so at 1-month follow-up. Working memory training had no effects on numerical knowledge. Extending some of this work to clinical populations, a study by Cheng et al in this issue investigated the specific mechanisms that can account for previously reported improvements in arithmetic learning following numerositybased training in children with developmental dyscalculia. To do so, they focused specifically on the role of visual form. Eighty-eight children with developmental dyscalculia were assigned to an experimental or a control training group (English dictation training) and trained over a period of 8 days. It was found that compared to a control group the experimental group significantly improved on subtraction, numerosity comparison as well as geometry.

These studies demonstrate the potential of careful and hypothesis-driven work focussing on specific training mechanisms derived a priori. By contrast, the study by Jones et al tested the role of domain-general mechanisms in supporting far transfer. As mentioned above, reports of working memory training on academic achievement have been mixed. One of the main criticisms of working memory interventions has been that working memory trainings might primarily promote the acquisition of highly task-specific strategies and that children require guidance on how these can be implemented in new contexts. Jones et al combined classical working memory training with a metacognitive strategy training and tested its effects on assessments of working memory as well as reading and maths, before and after training as well as at three-month follow-up. It was found that compared to an active control group such combined training ingredients led to improvements in maths, which was sustained at follow-up. These findings support the value of promoting insight to foster transfer of learning across domains.

\section{Neural mechanisms}

Given the functions that specific brain regions perform, studying brain changes associated with cognitive trainings can offer insight into the underlying neural mechanisms and the level at which change occurs. The study by Delande is one of the few studies to look at changes in brain structure following inhibitory control training in development. As mentioned above, the most commonly trained executive function has been working memory. This is in large part due to the 
straightforward way in which working memory tasks lend themselves to perform such a function. How to train inhibitory control has been harder to infer, yet recent attempts have been promising (Berkman, Kahn, \& Merchant, 2014). Delande et al studied the effects of a 5-week computerized inhibitory control training in 123 children and adolescents on brain regions associated with inhibition and compared this with a knowledge- and vocabulary-based training group. It was found that inhibitory control training led to improvements in inhibitory control in children but not adolescents. Further, among children only boys showed this effect. Analyses of brain structure showed significant alterations as a function of training in brain regions known to support inhibitory control. These findings are highly promising, and point to the complex nature of gender specific developmental processes that might underlie training-related gains in cognitive training.

\section{Novel techniques}

Sophisticated analytic techniques can provide crucial insights into how training and transfer effects arise. Structural equation models for instance allow studying transfer effects not at the level of specific tasks but at the level of underlying latent constructs. In an impressive language intervention, Melby-Levrag et al devised a 30 -week programme comprised of 90 sessions. Over this period participants engaged in two group sessions and one individual session per week; these aimed to train children's oral language as well as vocabulary skills, narrative skills and active listening skills. A control group followed their ordinary preschool program, which also involved some reading and language activities. Importantly, the extent of transfer was measured by how comparable the outcome measures were to the trained material (i.e. near transfer: taught words; intermediate transfer: sentences that contained taught words; far transfer: a latent factor of six general language measures without the taught vocabulary). The intervention had an effect on both near and intermediate transfer measures and only on the far transfer measures which affected language production. A mediation model showed that the far transfer effects were mediated entirely through the trained words. These findings suggest that far transfer is possible within the same domain, something which can be explained by current theories on far transfer effects (Taatgen, 2013).

Other tools to address mechanistic questions related to cognitive training and transfer include multivariate techniques and machine learning algorithms. One question that has received relatively little attention is on the role of individual differences in the size of training and transfer effects. Extant theories include compensation accounts (i.e. those with high baseline scores have 
less to gain) and magnification accounts (i.e. those with high baseline scores have more to gain), yet these views are unlikely to fully account for the complexity of training differences across individuals. The study by Rennie et al., used machine learning techniques to identify how and why children respond so differently to working memory training. Relying on multivariate data and using artificial neural networks, the authors studied how task representations change following training and which individual differences might predict such changes in task representations. Drawing on three large data sets $\left(N_{1}=526 ; N_{2}=90 ; N_{3}=179\right)$ using Cogmed working memory training, the authors found relationships between performance on different tasks to change as a function of training. Further, they identified subgroups of participants characterized by how well they did on the combination of tasks and found that training-related changes were different for these subgroups. Participants with higher fluid reasoning scores ended up gaining most from the training. This study highlights the huge potential of multivariate statistics and machine learning in addressing some of the most important questions in the field of cognitive training.

\section{Unchartered domains}

As stated above, most cognitive interventions aim to improve academic achievement or intelligence. Therefore, most of what we know in this field is limited to a relatively circumscribed set of transfer domains. Branching out to other, but no less important fields of cognitive functioning remains an important task. For instance, there is accumulating evidence that poor executive functions pose a risk for the development and onset of a range of mental health disorders (Johnson, 2012). Attentional control has been argued to play a key role in vulnerability to depression and anxiety. In this issue, Beloe and Derakshan use an adaptive dual n-back task as working memory training in adolescents, a developmental period where many mental health disorders commonly present. Training was given to 254 10-18 year olds, with an adaptive version of the n-back task for the experimental and a non-adaptive version for the control group. Selfreported depression and anxiety were lower following training in the intention-to-treat sample but only for the adaptive training group. These findings are promising for either preventing or intervening on internalising symptoms in developmental groups at risk for mental health disorders.

Frankenhuis et al turn the logic of interventions on its head in a highly innovative approach, looking at the potentially beneficial effects of harsh environments on circumscribed cognitive abilities, namely perceiving and remembering social hierarchies. In a study that looked at the effects of current as well as childhood exposure to violence, the authors found what they call "hidden talents". Current levels of personal involvement of violence were associated with better 
memory for dominance relations. This finding highlights the importance of understanding the developing system as adaptive to specific circumstances, which can confer both deficits as well as advantages. This study is also innovative by taking real life experiences (as opposed to an artificial training context) and investigating how these can shape cognitive functioning across a broad range of other domains. It is critical for the field of cognitive training to reflect on the curious fact that context may play a crucial role in whether transfer occurs or not. Ultimately, the field of cognitive training will need bridge the highly experimentally controlled interventions and more contextualised and ecologically valid experiences.

\section{Conclusion}

This Special Issue comprises a series of highly innovative research articles that each contribute to our understanding of mechanisms of cognitive training and transfer effects in developmental populations. From identifying the true relationship between two variables (a critical feature of any training study; Altarelli et al), to targeting highly specific training mechanisms (Johann \& Karbach, Gilligan, Azarzahu, Cheng, Ramani, Jones) and pinpointing underlying neural mechanisms (Delande), this set of articles demonstrate that cognitive trainings lead to tangible and durable transfer across different domains. More importantly, they show that a systematic programme of establishing a mechanistic understanding of training and transfer effects can be leveraged to ensure that interventions can potentially be applied beyond lab settings. The use of novel methods to implement training paradigms (Johann and Karbach) and to analyse training-related change (Rennie; Melby-Levag; and in fact the training process itself) is critical in furthering this endeavour. Lastly, the necessity to extend beyond domains beyond academic attainment, particularly mental (Beloe; Frankenhuis) but also physical health will be crucial to understand the extent of training effects and critically evaluate claims whether experiences generalize to other contexts or not. This set of empirical papers also raises the bar in terms of how training studies are carried out. Naturally, outstanding questions remain. Does learning generalize by means of the same mechanism independent of the learning domain? How does training and transfer change with age? The data to address these questions is difficult to come by, but longitudinal 
studies with a large age range and different training components are best positioned to address these. Finally, the field of cognitive training intersects between basic and applied science. The mere demonstrations of transfer have sometimes precluded the necessity for an overarching theory that can account for such effects. While theories of transfer effects exist (Taatgen, 2013), these need to be harnessed to make clear and falsifiable predictions when transfer would be expected. In sum, wholesale claims that cognitive training does not enhance processing in other cognitive domains are premature and the rigorous and hypothesis-driven research exemplified in this Special Issue is our best bet at establishing when transfer can and when it cannot be expected to occur.

\section{References:}

Berkman, E. T., Kahn, L. E., \& Merchant, J. S. (2014). Training-Induced Changes in Inhibitory Control Network Activity. Journal of Neuroscience, 34(1), 149-157.

Best, J. R., Miller, P. H., \& Naglieri, J. A. (2011). Relations between executive function and academic achievement from ages 5 to 17 in a large, representative national sample. Learning and Individual Differences, 21(4), 327-336.

Diamond, \& Lee. (2011). Interventions Shown to Aid Executive Function Development in Children 4 to 12 Years Old. Science, 333, 959-964.

Green, C. S., Bavelier, D., Kramer, A. F., Vinogradov, S., Ansorge, U., Ball, K., et al. (2019). Improving methodological standards in behavioral interventions for cognitive enhancement. Journal of Cognitive Neuroscience, 3(1), 2-29.

Johnson, M. H. (2011). Interactive specialization: a domain-general framework for human functional brain development? Dev Cogn Neurosci, 1(1), 7-21.

Johnson, M. H. (2012). Executive function and developmental disorders: the flip side of the coin. Trends Cogn Sci, 16(9), 454-457. 
Karbach, J., \& Verhaeghen, P. (2014). Making working memory work: a meta-analysis of executive-control and working memory training in older adults. Psychol Sci, 25(11), 20272037.

Karmiloff-Smith, A. (1998). Development itself is the key to understanding developmental disorders. Trends in Cognitive Sciences, 2(10), 389-398.

Sala, G., \& Gobet, F. (2017). Working Memory Training in Typically Developing Children: A MetaAnalysis of the Available Evidence. Developmental Psychology, 53(4), 671-685.

Sala, G., \& Gobet, F. (2019). Cognitive Training Does Not Enhance General Cognition. Trends Cogn Sci, 23(1), 9-20.

Schellenberg, E. G. (2005). Music and cognitive abilities. Current Directions in Psychological Science, 14(6), 317-320.

Simons, D. J., Boot, W. R., Charness, N., Gathercole, S. E., Chabris, C. F., Hambrick, D. Z., et al. (2016). Do "Brain-Training" Programs Work? Psychol Sci Public Interest, 17(3), 103-186.

Strobach, T., \& Karbach, J. (2016). Cognitive Training. Cham: Springer.

Taatgen, N. A. (2013). The Nature and Transfer of Cognitive Skills. Psychological Review, 120(3), 439-471.

Titz, \& Karbach, J. (2014). Working memory and executive functions: effects of training on academic achievement. Psychol Res.

Uttal, D. H., Meadow, N. G., Tipton, E., Hand, L. L., Alden, A. R., Warren, C., et al. (2013). The Malleability of Spatial Skills: A Meta-Analysis of Training Studies. Psychological Bulletin, 139(2), 352-402.

Wass, S., Scerif, G., \& Johnson, M. H. (2012). Training attentional control and working memory Is younger, better? Developmental Review, 32(4), 360-387. 